\title{
Empfehlungen zur Struktur der Betreuung von Erwachsenen mit angeborenen Herzfehlern (EMAH Patienten) in Österreich - Update 2011
}

\author{
Harald M. Gabriel · Helmut Baumgartner · Andreas Gamillscheg · Igor Knez • \\ Robert Maier · Ina Michel-Behnke - Bert Nagel · Raphael Rosenhek · \\ Jörg Ingolf Stein · Gregor Wollenek · Johannes Mair
}

Eingegangen: 29. Oktober 2013 / Online publiziert: 17. Dezember 2013

(C) Die Autor(en) 2013. Dieser Artikel ist auf Springerlink.com mit Open Access verfügbar.

\section{Einleitung}

Erwachsene Patienten/innen mit angeborenen Herzfehlern (EMAH) stellen im Gesamtspektrum der von „Erwachsenenkardiologen/innen“ zu betreuenden Patienten/innen eine kleine Gruppe dar und doch ist von einer Patientenanzahl von ca. 350.000 Personen in den D-A-CH (Deutschland-Österreich-Schweiz)-Ländern auszugehen. Die Probleme dieser PatientInnen unterscheiden sich in vielen Dingen grundlegend von jenen der sonst in der Erwachsenkardiologie zu betreuenden Patienten/innen. Eine adäquate Behandlung setzt daher besondere Kenntnisse und Erfahrungen des zuständigen

Für die Arbeitsgruppe „Angeborene und Erworbene Herzfehler im Jugend- und Erwachsenenalter“ der Österreichischen Kardiologischen Gesellschaft

H. M. Gabriel $(\bowtie) \cdot$ R. Rosenhek

Universitätsklinik für Innere Medizin II,

Klinische Abteilung für Kardiologie,

Medizinische Universität Wien, Währinger Gürtel 18-20,

1090 Wien, Österreich

E-Mail: harald.gabriel@meduniwien.ac.at

\section{H. Baumgartner}

Klinik für angeborene und erworbene Herzfehler,

Universitätsklinikum Münster, Münster, Deutschland

A. Gamillscheg · B. Nagel

Universitätsklinik für Kinder- und Jugendheilkunde,

Klinische Abteilung für Pädiatrische Kardiologie,

Medizinische Universität Graz, Graz, Österreich

I. Knez

Universitätsklinik für Chirurgie, Klinische Abteilung für

Herzchirurgie, Medizinische Universität Graz, Graz, Österreich

R. Maier

Universitätsklinik für Innere Medizin,

Klinische Abteilung für Kardiologie,

Medizinische Universität Graz, Graz, Österreich
Arztes voraus. Eine wesentliche Voraussetzung dafür, eine entsprechende Kompetenz aufzubauen und aufrechtzuerhalten, ist vor allem die ständige Beschäftigung mit dieser Materie und die regelmäßige Betreuung einer hohen Patientenzahl. Detaillierte aktuelle Behandlungsrichtlinien der D-A-CH AG für Erwachsene Patienten mit angeborenen Herzfehlern wurden unlängst auch im J Kardiol publiziert [1], aktuelle Richtlinien der amerikanischen und europäischen Fachgesellschaften liegen vor [2, 3]. Daher konzentrieren sich diese Empfehlungen auf die Strukturen der Versorgungseinrichtungen und die erforderlichen Qualifikationen der Leiter der Versorgungseinrichtungen für EMAH Patienten/innen in Österreich.
I. Michel-Behnke

Universitätsklinik für Kinder- und Jugendheilkunde,

Klinische Abteilung für Pädiatrische Kardiologie,

Medizinische Universität Wien, Wien, Österreich

\section{J. I. Stein}

Universitätsklinik für Pädiatrie III, Kardiologie,

Pulmologie, Allergologie, Cystische Fibrose,

Medizinische Universität Innsbruck, Innsbruck, Österreich

\section{G. Wollenek}

Universitätsklinik für Chirurgie, Klinische Abteilung für Herzchirurgie, Medizinische Universität Wien, Wien, Österreich

J. Mair

Universitätsklinik für Innere Medizin III,

Klinische Abteilung für Kardiologie,

Medizinische Universität Innsbruck, Innsbruck, Österreich 
Nach internationalen Erfahrungen kann eine optimale Versorgung dieser Patientengruppe daher nur durch besonders dafür ausgerichtete Zentren für angeborene Herzfehler im Erwachsenenalter gesichert werden, die mit den jeweiligen betreuenden Kardiologen/ innen bzw. Internen Abteilungen vor Ort entsprechend eng zusammenarbeiten. Ein Ziel der Arbeitsgruppe für angeborene und erworbene Herzfehler im Erwachsenenalter der Österreichischen Kardiologischen Gesellschaft ist es daher, auch in Österreich aktuelle Empfehlungen für entsprechende Strukturen der regionalen und überregionalen Versorgung auszuarbeiten und diese Richtlinien unter Internisten/innen mit kardiologischem Schwerpunkt bzw. Kardiologen/innen zu verbreiten. Dieses Update der erstmals im Jahre 2001 publizierten Österreichischen Richtlinien lehnt sich an international anerkannte Vorschläge an insbesondere an die rezenten deutschen Empfehlungen [3-7] mit Adaptationen an die Situation in Österreich wo es notwendig war. Dabei erscheint eine Differenzierung in regionale und überregionale Versorgung der EMAH Patienten als sinnvoll.

\section{Kriterien eines überregionalen Zentrums für angeborene Herzfehler im Erwachsenenalter}

Prinzipiell ist hervorzuheben, dass die Betreuung eine enge Zusammenarbeit zwischen Kinder- und Erwachsenenkardiologen/innen zur Vorraussetzung hat und in einem Zentrum angesiedelt sein soll, das alle medizinischen Abteilungen zur Versorgung von Erwachsenen anbieten kann [3]. Ein solches überregionales Zentrum zeichnet sich weiters durch eine eigene Abteilung für Kardiologie, Kinderkardiologie (beide mit erfahrenen interventionellen Kardiologen/innen, nach Möglichkeit inklusive Elektrophysiologie) und Herzchirurgie und Kardioanästhesie und Intensivmedizinern/innen mit Erfahrung in der operativen bzw. postoperativen Versorgung von kongenitalen Vitien aus. Zusätzlich sollen alle modernen Methoden der kardiovaskulären Bildgebung zur Verfügung stehen (z. B. kardiales MRI und CT mit state-of-the-art Technologie Geräten).

\section{Aufgaben des überregionalen Zentrums}

- Optimierung der Versorgung von erwachsenen Patienten/innen mit allen Formen von angeborenen Herzfehlern unter Berücksichtigung der Besonderheiten in Hämodynamik und Anatomie, die sich substantiell von den regulären kardialen Erkrankungen im Erwachsenenalter unterscheidet, um Fehleinschätzung und -behandlungen zu vermeiden.

- Vereinigung aller speziellen Ressourcen, die für die Versorgung von EMAH Patienten erforderlich sind.

- Sicherstellung einer hohen EMAH Patientenzahl, die ein entsprechendes Training sowie das Aufrechterhalten einer entsprechenden Kompetenz und spezieller Fähigkeiten in der Behandlung von EMAH Patienten ermöglicht.
- Wissenschaftliche Aufarbeitung dieser Patientengruppe und entsprechende Verbesserung unseres Wissens für zukünftige Behandlungen.

- Angebot von entsprechender Fortbildung für die in die Betreuung von EMAH Patienten eingebundenen Internisten/innen, Kardiologen/innen und Chirurgen/innen, Kardioanästhesisten/innen, Gynäkologen/innen.

- Angebot von Informationsmöglichkeiten für Patienten/innen und Ärzte/innen bzw. der Möglichkeit, entsprechende Expertenmeinungen einzuholen.

- Unterstützung von Patientenselbsthilfegruppen.

- Ansprechpartner für Ämter und Behörden.

\section{Erforderliche Ressourcen eines überregionales EMAH Zentrums}

A. Personal:

Voraussetzung für das Personal ist eine besondere Erfahrung und eine besondere Ausbildung in der Betreuung von EMAH Patienten, wobei zur Verfügung stehen sollte:

- zumindest 2 Erwachsenenkardiologen/innen und 2 Kinderkardiologen innen mit spezieller Erfahrung auf dem Gebiet angeborener Herzfehler im Erwachsenenalter

- zumindest 2 Chirurgen/innen mit spezieller Erfahrung auf dem Gebiet der angeborenen Herzfehler

- ein(e) Transplantationschirurg/in bzw. eine fix bestehende Zusammenarbeit mit einem Transplantationszentrum

- ein Elektrophysiologe/in mit Erfahrung im Bereich der angeborenen Herzfehler bzw. Kooperation mit internationalem Zentrum für Patienten/innen mit komplexen angeborenen Herzfehlern

- Spezialist/in mit Erfahrung in Implantation und Nachsorge von Schrittmachern, ICD und biventrikulären Schrittmachern

- zumindest ein Kardiologe/in/Kinderkardiologe/in mit nachgewiesener Erfahrung mit nichtkoronaren Katheterinterventionen

- zumindest 2 Echokardiographeure/innen mit speziellen Kenntnissen im Bereich der angeborenen Herzfehler

- zumindest 2 Herzanästhesisten/innen mit Erfahrung in der Anästhesie von Patienten mit angeborenen Herzfehlern

- Zumindest ein(e) Radiologe/in mit Erfahrung in der Anwendung von CT und MRI bei EMAH Patienten/ innen.

- ein Sozialarbeiter/in bzw. -berater/in

- ein Psychologe/in

- Schwestern/Pfleger mit spezieller Erfahrung mit Patienten mit angeborenen Herzfehlern

- Ein Herzpathologe/in mit Erfahrung auf diesem Gebiet der angeborenen Herzfehler

- Ein Facharzt/in für Gynäkologie und Geburtshilfe als fixe(r) Ansprechpartner/in im Team 
- Schließlich sollten am Zentrum alle Spezialgebiete der Inneren Medizin und alle Sonderfächer inklusive Humangenetik, Psychiatrie und Medizinische Psychologie vertreten sein.

B. Die folgenden apparativen Voraussetzungen und Funktionseinheiten sollten vorhanden sein:

- Echokardiographie einschließlich transösophagealer Echokardiographie (inkl. Intraoperativer TEE), Streßechokardiographie

- Herzkatheterlabor für diagnostische und interventionelle Prozeduren

- Elektrophysiologielabor mit Möglichkeiten zu Ablation

- Herzoperationssaal

- Extrakorporale Kreislaufunterstützungssysteme (ECMO, Assist-Device)

- Möglichkeiten zur stationären Betreuung mit Intensivstation, Intermediate Care und Normalstation

- Komplette Palette der bildgebenden Verfahren (kardiovaskuläre Radiologie, Computertomographie, Magnetresonanz)

- Nuklearmedizin

- Schrittmacher-/ICD/CRT-Ambulanz

- Langzeit-EKG, Event-Recorder, externe und implantierbare Look-Recorder

- Ergometrie

- Lungenfunktionslabor/Spiroergometrie

- Herzpathologie

- Datenbanksystem für die Patientendokumentation und zur Qualitätskontrolle

\section{Funktionen des überregionalen Zentrums}

- Optimale Versorgung von Erwachsenen mit angeborenen Herzfehlern

- Geordnete Übernahme von Patienten/innen aus der Kinderkardiologie in die Erwachsenenkardiologieabteilung oder gemeinsame Weiterbetreuung im Rahmen einer Spezialambulanz in Zusammenarbeit mit einem Kardiologen/in

- Regelmäßige gemeinsame Konferenzen mit Kardiologen/innen, Kinderkardiologen/innen, Herzchirurgen/innen und weiteren Spezialisten/innen zur Entscheidungsfindung für Behandlungsstrategien

- Sicherung einer adäquaten und zeitgerechten Kommunikation mit zuweisenden Ärzten/innen bzw. kooperierenden Abteilungen

- Maßnahmen zur Qualitätskontrolle im Zentrum selbst und Zusammenarbeit mit andern EMAH Zentren zur Qualitätssicherung und ständigen Verbesserung der Patientenversorgung

- Errichtung von Trainingsprogrammen für Kardiologen/innen, Kinderkardiologen/innen und Chirurgen/ innen

- Datenbank über alle betreuten Patienten/innen
- Aufbau und Erhaltung von Strukturen, die eine interdisziplinäre Versorgung von EMAH Patientinnen während der Schwangerschaft und zur Entbindung erlauben sowie zur Betreuung von Hochrisiko-Patienten bei nichtherzchirurgischen Operationen

- Kooperation mit Patientenorganisationen und Rehabilitationseinrichtungen

\section{Regionale Versorgung von EMAH Patienten}

In einer Klinikambulanz oder ambulanten Einrichtung zur regionalen EMAH Patientenversorgung sollte mindestens 1 Kardiologe/in in Zusammenarbeit mit einem Kinderkardiologen/in (falls vorhanden) mit speziellen Fähigkeiten, speziellem Training und spezieller Erfahrung in der Versorgung von EMAH Patienten vorhanden sein. Weiters ist besondere Erfahrung in der Echokardiographie von angeborenen Herzfehlern Voraussetzung. Die strukturellen Komponenten eines regionalen Zentrums richten sich nach den lokalen Ressourcen, Möglichkeiten und Notwendigkeiten, wobei ein hochwertiges Echokardiographiegerät, eine Ergometrieeinheit, das $24 \mathrm{~h}$ Blutdruckmonitoring und das Langzeit-EKG zur Grundausstattung gehören. Prinzipiell können in einem solchen Zentrum Erwachsene mit allen Formen von kongenitalen Herzfehlern basisversorgt werden, wobei durch die Zusammenarbeit mit einem überregionalen Zentrum die lokal nicht vorhandenen diagnostischen und therapeutischen Möglichkeiten kompensiert werden können.

Bei größerer geographischer Distanz zu einem überregionalen EMAH Zentrum ist der Aufbau einer regionalen EMAH Patienten/innen Versorgung im jeweiligem Bundesland anzustreben. Die enge Zusammenarbeit der Ärzte der überregionalen Zentren mit den lokal die EMAH Patienten betreuenden Ärzten/innen ist für eine gute Betreuung essentiell.

\section{Erfordernisse für die Leitung eines} klinikgebunden oder ambulanten Zentrums für angeborene Herzfehler im Erwachsenenalter

Diese Qualifikationen lehnen sich an die Deutschen Empfehlungen [7] zum Erwerb der Zusatzqualifikation „Erwachsene mit angeborenen Herzfehlern“ (EMAH) an. Für neuauszubildende EMAH Kardiologen/innen empfehlen wir folgende Spezialausbildung:

Dauer der zusätzlichen Weiterbildung. Die Weiterbildung in dieser Subspezialität erstreckt sich über 18 Monate, wobei ausbildungsinhaltskonforme Zeiten (siehe unten) der Additivfacharztausbildung in Kardiologie bzw. Kinderkardiologie berücksichtigt werden können. Die Weiterbildung kann im Inland oder Ausland erfolgen. 
Die EMAH-Weiterbildung für ErwachsenenkardiologInnen umfasst:

- eine 6-monatige Tätigkeit an einem kinderkardiologischen Zentrum,

- eine 6-monatige Tätigkeit an einem überregionalen EMAH-Zentrum,

- die restlichen 6 Monate können entweder in einem regionalen oder überregionalen EMAH-Zentrum absolviert werden.

\section{Die EMAH-Weiterbildung für KinderkardiologInnen} umfasst:

- eine 6-monatige Tätigkeit in einer Internistischen Abteilung mit Schwerpunkt Kardiologie,

- eine 6-monatige Tätigkeit an einem überregionalen EMAH-Zentrum,

- die restlichen 6 Monate können entweder in einem regionalen oder überregionalen EMAH-Zentrum absolviert werden.

\section{Indikation zur Zuweisung in ein regionales oder} überregionales EMAH Zentrum

Prinzipiell sollen alle Patienten mit angeborenen Herzfehlern an einem EMAH Zentrum betreut werden, ausgenommen davon sind:

- Aortenklappenerkrankungen (ausgenommen komplexe Fehlbildungen sowie in der Kindheit chirurgisch oder katheterinterventionell behandelte Patienten/innen mit Aortenstenose)

- komplett korrigierte Fehlmündung von Lungenvenen

- korrigierte Pulmonalstenose ohne bedeutsame Restgradienten und bedeutsame Pulmonalinsuffizienz

- leicht- bis mittelgradige Pulmonalstenose

- Mitralklappenerkrankungen (ausgenommen Parachute- Klappe und ähnliche Anomalien)

- Zustand nach komplettem Verschluss eines Ventrikelseptumdefektes (ohne Restshunt) bei sonst unauffälligem Befund

- Zustand nach Komplettverschluss eines Ductus arteriosus

- Zustand nach Verschluss eines Vorhofseptumdefektes (ohne Restdefekt)

\section{Interessenkonflikt}

Es besteht kein Interessenkonflikt.

\section{Open Access}

Dieser Artikel unterliegt den Bedingungen der Creative Commons Attribution Noncommercial License. Dadurch sind die nichtkommerzielle Nutzung, Verteilung, und Reproduktion erlaubt, sofern der/die Originalautor/en und die Quelle angegeben sind.

\section{Anhang}

Modifiziert nach [8]

\begin{tabular}{|l|}
\hline Anforderungen an ein überregionales EMAH Zentrum \\
\hline Kinderkardiologie \\
\hline Kardiologie \\
\hline Kooperation mit einer Rhythmologie mit elektrophysiologischem Labor \\
\hline Herzchirurgie für AHF \\
\hline Kardio-Anästhesiologie \\
\hline Intensivtherapie \\
\hline Radiologie (MRT, CT) \\
\hline Gynäkologie und Geburtshilfe \\
\hline Innere Medizin \\
\hline Kooperation mit einer Transplantationseinheit \\
\hline Humangenetik \\
\hline Psychologie \\
\hline Sozialmedizin \\
\hline Fach-Schwestern/-Pfleger \\
\hline Kooperation mit Patientenorganisationen \\
\hline Kooperation mit Rehabilitationseinrichtungen \\
\hline
\end{tabular}

\section{Referenzen}

1. Schmaltz AA, Bauer U, Baumgartner $\mathrm{H}$, et al. Medizinische Leitlinie zur Behandlung von Erwachsenen mit angeborenen Herzfehlern (EMAH) der deutsch-österreichischschweizerischen kardiologischen Fachgesellschaften. J Kardiol. 2010;17:(Pre-Publishing Online).

2. Warnes CAW, Williams RG, Bashore TM, et al. ACC/AHA guidelines for the management of adults with congenital heart disease. Circulation. 2008;118:e714-833.

3. Baumgartner H, Bonhoeffer P, De Groot NMS, et al. ESC guidelines fort he management of grown-up congenital heart disease (new version 2010). Eur Heart J. 2010;31:2915-57.

4. Foster E, Graham TP, Driscoll DJ, et al. Task force 2: special Health care needs of adults with congenital heart disease. JACC. 2001;37:1176-83.

5. Child JS, Collins-Nakai RL, Alpert JS, et al. Task force 3: workforce description and educational requirements for the care of adults with congenital heart disease. JACC. 2001;37:1183-7.

6. Landzberg MJ, Murphy DJ, Davidson WR, et al. Task force 4: organization of delivery systems for adults with congenital heart disease. JACC. 2001;37:1187-93.

7. Hess J, Bauer U, Haan de F, et al. Empfehlungen für Erwachsenen- und Kinderkardiologen zum Erwerb der ZusatzQualifikation „Erwachsene mit angeborenen Herzfehlern“ (EMAH). Clin Res Cardiol. 2007;2(Suppl.):19-26.

8. Kaemmerer H, Breithart G. Empfehlungen zur Qualitätsverbesserung der interdisziplinären Versorgung von Erwachsenen mit angeborenen Herzfehlern (EMAH). Clin Res Cardiol. 2006;95(Suppl. 4):76-84. 